\title{
A program for constructing SVT tests: An alternative way of assessing text comprehension
}

\author{
JEFFREY J. WALCZYK and JAMES M. ROYER \\ University of Massachusetts, Amherst, Massachusetts
}

\begin{abstract}
The sentence verification technique (SVT), developed by Royer, Hastings, and Hook (1979), provides developmental and educational researchers with an alternative way of assessing text com prehension based on all of the information contained in a passage. Students must decide if test sentences have the same meaning as sentences that appeared in an original passage. The program described here automates the more laborious aspects of constructing SVT tests. The user must create a passage using a word processor. The program will prompt the user for information as needed. Finally, printable text files (SVT test and answer key) are generated. The program is written in BASIC for IBM and IBM-compatible computers.
\end{abstract}

The sentence verification technique (SVT), introduced by Royer, Hastings, and Hook (1979), provides educational and developmental researchers with an alternative way of assessing comprehension of text. Whereas traditional methods typically require the reader to respond to literal and inferential multiple-choice items after reading a passage, SVT tests require students to determine whether test sentences accurately represent the meaning of corresponding sentences from an original passage.

A problem with traditional measures is that, in addition to measuring comprehension, they may also be measuring other abilities such as intelligence (Johnston, 1984). SVT tests, on the other hand, do not measure inferential reasoning. Rather, they measure the extent to which readers have been successful in acquiring the meaning of a test passage. Thus, they may represent a purer measure of text comprehension.

SVT tests have been shown to have desirable psychometric properties. They are sensitive to differences in text readability and in skill level (Royer \& Hambleton, 1983; Royer et al., 1979). They predict learning performance in college courses (Royer, Abranovic, \& Sinatra, 1987), and they measure passage, not just sentence, comprehension (Royer, Lynch, Hambleton, \& Bulgarelli, 1984).

\section{How SVT Tests are Constructed Manually}

SVT tests are constructed by means of selecting one of three types of test sentences to represent each sentence appearing in an original passage. Thus, it is desirable that the number of sentences in the original passage be evenly divisible by 3 ; there should be, for example, 9,12 , or 15 of them. The three types of test sentences are called originals, meaning changes, and paraphrases. The origi-

Correspondence may be addressed to Jeffrey J. Walczyk, Department of Psychology, University of Massachusetts, Amherst, MA 01003. nals are verbatum copies of their corresponding passage sentences. The meaning changes contain many of the same words and have the same syntactic structure as their corresponding passage sentences, but one or two of the words are changed in them so that they have distinctly different meanings. The paraphrases express the same meanings as their corresponding passage sentences, but in different words. Finally, a group of distractor sentences, equal to the number of sentences in the other groups, must be constructed. These distractors have meanings consistent with the theme of the passage but do not correspond to any sentences that actually occur in the passage.

The order of the test sentences should be randomized on an SVT test, under the constraints that (1) sentences occurring in the first half of the passage occur in the first half of the test, and (2) an equal number of originals, meaning changes, paraphrases, and distractors occur in each half of the test. For a more detailed but nontechnical account of how to construct SVT tests, see Royer, Greene, and Sinatra (1987).

\section{Motivation for the SVT \\ Test-Construction Program}

The procedure above, however, because it is normally performed manually, is both laborious and prone to error. The SVT test-construction program described here improves things by automating many of the steps. In particular, the program will read an original passage, parse it into sentences, and randomly assign sentences to SVT item types, observing the constraints above. Once the test items have been entered, the computer will synthesize them into an SVT test complete with directions. An answer key is also generated.

\section{Language and Devices Needed}

The program is written in BASIC and is available only for MS-DOS (IBM or IBM-compatible) machines. BASIC 
or BASICA, provided with most machines, is suitable for running the program. It is also desirable to have a printer available for hard copy output.

\section{Running the Program}

The program accepts as input a text file (the original passage). The file can be created with any editor or word processing system, and it must be stored as a standard $A S C I I$ file. After the program parses the sentences, either the user can assign the sentences to SVT item types, or the computer can randomly determine this. The program assigns each sentence to a string variable. Since BASIC limits the size of string variables, no sentence should be more than 255 characters long; otherwise an error condition will occur. Next, the user must develop and enter meaning-change and paraphrase items, given the corresponding original sentences. Finally, the distractors are entered. The computer then constructs the SVT test. The generated test and answer key are ASCII text files that can be printed or modified with a word processor. The order of test items is randomized, subject to the constraints defined above.

The program is user friendly. The user is provided with detailed instructions at every point of execution, which clarify what information must be entered or precisely what the computer is doing. The user is given ample opportunity to correct any mistakes. Finally, for BASIC programmers who might wish to modify the program for their own purposes, internal documentation is provided.

\section{Limitations}

The user must have BASIC or BASICA on the same floppy or in the same subdirectory as the program. In addition, the floppy or subdirectory must not be write- protected. That is, the program must be free to write text files to disk. The program can be called up and run from MS-DOS level by typing BASIC SVT, or BASICA SVT, and then pressing the RETURN or ENTER key. Once the program is through executing, the user is returned to the operating system level.

\section{Program Availability}

The program will be provided on a 5.25-in. floppy for $\$ 8$ to cover costs. Simply send a check and return address to Jeffrey J. Walczyk, at the following address: Department of Psychology, University of Massachusetts at Amherst, Amherst, MA 01003.

\section{REFERENCES}

Johnston, P. (1984). Prior knowledge and reading comprehension test bias. Reading Research Quarterly, 19, 219-239.

Royer, J. M., Abranovic, W. A., \& Sinatra, G. M. (1987). Using entering reading performance as a predictor of course performance in college classes. Journal of Educational Psychology, 79, 19-26.

Royer, J. M., Greene, B. A., \& Sinatra, G. M. (1987). The sentence verification technique: A practical procedure for testing comprehension. Journal of Reading, 30, 414-423.

Royer, J. M., \& Hambleton, R. K. (1983). Normative study of 50 reading comprehension passages that use the sentence verification technique. Unpublished manuscript, University of Massachusetts, Amherst, MA.

RoYeR, J. M., Hastings, C. N., \& Hook, C. (1979). A sentence verification technique for measuring reading comprehension. Journal of Reading Behavior, 11, 355-363.

Royer, J. M., Lynch, D. J., Hambleton, R. K., \& Bulgarell, C. (1984). Using the sentence verification technique to assess the comprehension of technical text as a function of subject matter expertise. American Educational Research Journal, 21, 839-869.

(Manuscript received January 5, 1989; revision accepted for publication March 30, 1989.) 